\title{
MINIMAL CONDITIONS ON CLIFFORD SEMIGROUP CONGRUENCES
}

\author{
M. EL-GHALI M. ABDALLAH, L. N. GAB-ALLA, AND SAYED K. M. ELAGAN
}

Received 6 April 2006; Accepted 6 April 2006

A known result in groups concerning the inheritance of minimal conditions on normal subgroups by subgroups with finite indexes is extended to semilattices of groups $[E(S)$, $\left.S_{e}, \varphi_{e, f}\right]$ with identities in which all $\varphi_{e, f}$ are epimorphisms (called $q$ partial groups). Formulation of this result in terms of $q$ congruences is also obtained.

Copyright (c) 2006 Hindawi Publishing Corporation. All rights reserved.

\section{Introduction and preliminaries}

A partial group as defined in [3] is a semigroup $S$ which satisfies the following axioms.

(i) For every $x \in S$, there exists a (necessarily unique) element $e_{x} \in S$, called the partial identity of $x$ such that $e_{x} x=x e_{x}=x$ and if $y x=x y=x$ then $e_{x} y=y e_{x}=e_{x}$.

(ii) For every $x \in S$, there exists a (necessarily unique) element $x^{-1} \in S$, called the partial inverse of $x$ such that $x x^{-1}=x^{-1} x=e_{x}$ and $e_{x} x^{-1}=x^{-1} e_{x}=x^{-1}$.

(iii) The operation $x \mapsto e_{x}$ is a homomorphism from $S$ into $S$, that is, $e_{x y}=e_{x} e_{y}$ for all $x, y \in S$, and the operation $x \mapsto x^{-1}$ is an antihomomorphism, that is, $(x y)^{-1}=$ $y^{-1} x^{-1}$ for all $x, y \in S$.

Consequently, a partial group is precisely a Clifford semigroup, that is, a regular semigroup with central idempotents, and this is characterized by Clifford structure theorem (see [4, Chapter IV, Theorem 2.1] or [5, Chapter II, Theorem 2]) as a (strong) semilattice of groups.

Thus, in particular, a partial group $S$ may be viewed as a strong semilattice of groups $S=\left[E(S) ; S_{e}, \varphi_{e, f}\right]$, where $S_{e}$ is the maximal subgroup of $S$ with identity $e(e \in E(S))$ and for $e \geq f$ in $E(S), \varphi_{e, f}$ is the homomorphism of groups $S_{e} \rightarrow S_{f}, x \mapsto x f$. Here $E(S)$ is the semilattice ( $e \geq f$ if and only if $e f=f$ ) of idempotents (partial identities) in $S$.

Let $S$ be a partial group. A subpartial group of $S$ is a subsemigroup of $S$ closed under the unary operations of $S$. A subpartial group of $S$ is wide (or full) if it contains $E(S)$.

A normal subpartial group of $S$ is a wide subpartial group $K$ of $S$ such that $x^{-1} K x \subset K$ for all $x \in S$. This notion is standard in the literature, and we refer in particular to [2] for the following consequences. 
Let $K$ be normal in $S, K_{e}$ is a normal subgroup of $S_{e}$ for every $e \in E(S)$.

$\rho_{K}=\left\{(x, y) \in S \times S: e_{x}=e_{y}\right.$ and $\left.x y^{-1} \in K\right\}$ is an idempotent-separating congruence on $S$ with $\operatorname{ker} \rho_{K}=K$.

Conversely, if $\rho$ is an idempotent-separating congruence on $S$, then $K=\operatorname{ker} \rho$ is a normal subpartial group of $S$ and $\rho_{K}=\rho$.

Let $\mathbf{N}(S)$ be the set of all normal subpartial groups of $S$, and let $\mathbf{C}^{\mathbf{i}}(S)$ be the set of all idempotent-separating congruences on $S$. For $N, M \in \mathbf{N}(S), N \vee M$ is the join $\langle N \bigcup M\rangle$, that is, the smallest normal subpartial group of $S$ containing $N \cup M$, and we have $N \vee$ $M=N M=M N$. For $\rho, \sigma \in \mathbf{C}^{\mathbf{i}}(S), \rho \vee \sigma$ is defined similarly, and we have $\rho \vee \sigma=\sigma \circ \rho=$ $\rho \circ \sigma$. Also $\rho_{N M}=\rho_{N} \circ \rho_{M}$ for all $N, M \in \mathbf{N}(S)$. Moreover, we have the following theorem.

Theorem 1.1 [2]. $(\mathbf{N}(S), \subset, \cap, \vee)$ and $\left(\mathbf{C}^{\mathbf{i}}(S), \subset, \cap, \vee\right)$ are complete modular lattices and $\mathbf{N}(S) \rightarrow \mathbf{C}^{\mathbf{i}}(S), N \mapsto \rho_{N}$ is a lattice isomorphism.

For sake of reference, cite from [1] the material required for the present work.

A q partial group is a partial group $S$ with identity 1 such that $\varphi_{1, e}: S_{1} \rightarrow S_{e}$ is an epimorphism (i.e., $S_{1} e=S_{e}$ ) for every $e \in E(S)$. This implies clearly that $\varphi_{e, f}: S_{e} \rightarrow S_{f}$ is an epimorphism for every e, $f \in E(S)$ with $e \geq f$.

THeORem 1.2 [1]. Let $S$ be a partial group with identity 1. Every wide subpartial group $K$ of $S$ contains a unique maximal $q$ subpartial group, denoted by $\mathbf{Q}(K)$, of S given by $(\mathbf{Q}(K))_{e}=$ $\operatorname{Im},\left.\varphi_{1, e}\right|_{K_{1}}=K_{1}$ e for all $e \in E(S)$. That is, $\mathbf{Q}(K)$ is a strong semilattice $E(S)$ of groups $K_{1} e$. Also $\mathbf{Q}(K)$ is non trivial (i.e., $\mathbf{Q}(K) \neq E(S)$ ) if and only if $K_{1}$ is a nontrivial group.

On the class of partial groups with identities, we have idempotent unary operation $S \mapsto \mathbf{Q}(S)$, where $\mathbf{Q}(S)$, defind as above, is the unique maximal $q$ subpartial group of $S$.

LEMMA 1.3. Let $S$ be a partial group with identity and let $\left\{S_{i}, i \in I\right\}$ be a family of wide subpartial groups of $S$. Then $\mathbf{Q}\left(\left\langle\bigcup_{i \in I} S_{i}\right\rangle\right)=\left\langle\bigcup_{i \in I} \mathbf{Q}\left(S_{i}\right)\right\rangle$. In particular, the join of any family of $q$ subpartial groups of $S$ is a $q$ subpartial group of $S$.

A normal subpartial group of a partial group $S$ with identity need not be a $q$ subpartial group, even if $S$ is a q partial group (e.g., [1, Example 4.4 ]). On the other hand, if $S$ is a $q$ partial group and $N$ is normal in $S$, then $\mathbf{Q}(N)$ is normal in $S$.

Let $S$ be a q partial group and let $\mathbf{Q N}(S)$ be the set of all $q$ normal subpartial groups of $S$.

LEMma 1.4. QN $(S)$ is a complete modular lattice with meet and join defined by

$$
\begin{aligned}
& M \wedge N=\mathbf{Q}(M \cap N), \\
& M \vee N=M N=\langle M \cup N\rangle .
\end{aligned}
$$

An idempotent-separating congruence $\rho$ on a partial group $S$ with identity 1 is called a $q$ congruence if for all $x, y \in S, x \rho y$ implies that $x=s y$ for some $s \in(\operatorname{ker} \rho)_{1}$, that is, for some $s \in S_{1}$ with $s \rho 1$.

Lemma 1.5. Let $S$ be a partial group with identity, and let $\rho$ be an idempotent-separating congruence on $S$. Then $\rho$ is a q congruence if and only if $K=\operatorname{ker} \rho$ is a q normal subpartial group of $S$. Equivalently for any subpartial group $K$ of $S, K$ is a $q$ normal subpartial group of $S$ if and only if $\rho_{K}$ is a $q$ congruence. 
Let $S$ be a partial group with identity 1 . The $\mathbf{Q}$ operation is defined on idempotentseparating congruences as follows:

$$
\mathbf{Q}(\rho)=\{(x, y): x \rho y, x=s y(\text { or } x=y s) \text { for some } s \in S \text { with } s \rho 1\} .
$$

Precisely, $\mathbf{Q}(\rho)$ is the unique maximal $q$ congruence on $S$ contained in $\rho$.

LEMmA 1.6. Let $S$ be a q partial group and let $\rho \in \mathbf{C}^{\mathbf{i}}(S)$. Then

$$
\mathbf{Q}(\rho)=\rho_{\mathbf{Q}(N)}, \quad \text { where } N=\operatorname{ker} \rho .
$$

Let $S$ be a $q$ partial group and let $\mathbf{Q C}(S)$ be the set of all $q$ congruences on $S$.

THEOREM 1.7. With join and meet given by $\rho \vee \sigma=\rho \circ \sigma=\sigma \circ \rho$ and $\rho \wedge \sigma=\mathbf{Q}(\rho \bigcap \sigma)$, $\mathbf{Q C}^{\mathrm{i}}(S)$ is a complete modular lattice and the mapping

$$
\mathbf{Q N}(S) \longrightarrow \mathbf{Q C}^{\mathbf{i}}(S), \quad N \longmapsto \rho_{N},
$$

is a lattice isomorphism.

As observed in [1, Section 1], q partial groups exist naturally as partial mappings from sets to groups. In the present paper, we consider minimal conditions on normal subpartial groups of partial groups with identities and we discuss the situations with which such conditions could be inherited by subpartial groups with finite indexes. Our principal goal is to extend the following known result in groups to appropriate classes of partial groups.

Theorem 1.8 [6, Theorem 3.1.8]. If a group $G$ satisfies $\min -n$ and $H$ is a subgroup of $G$ with finite index, then $H$ satisfies $\min -n$.

In this theorem, $\min -n$ is the minimal condition on normal subgroups. In other words a group $G$ is said to satisfy $\min -n$ if any nonempty family of normal subgroups has a minimal member, or equivalently there does not exist a proper descending chain $N_{1} \supset N_{2} \supset \cdots$ of normal subgroups of $G$. The proof of the above result depends on the notion of normal closures and cores in groups. Here we give the definitions and some properties (see [6, Section I.3]).

If $X$ is a nonempty subset of a group $G$, the normal closure of $X$ in $G$, denoted by $X^{G}$, is the intersection of all normal subgroups of $G$ which contains $X$. Dually, the core of $X$ in $G$, denoted by $X_{G}$, is the join of all normal subgroups of $G$ that contains by $X$. In other words, $X^{G}$ is the smallest normal subgroup of $G$ containing $X$, whereas $X_{G}$ is the largest one contained in $X$.

Fact 1.9. $X^{G}=\left\langle g^{-1} X g: g \in G\right\rangle$.

Fact 1.10. For any subgroup $H$ of $G, H_{G}=\bigcap_{g \in G} g^{-1} H g$.

For our work, we introduce in Section 2 normal closures and cores in partial groups, showing that if $S$ is a partial group and $\varnothing \neq X \subset S$, then $X^{S}$ is characterized as in Fact 1.9. If either $X \cap S_{e} \neq \varnothing$ for all $e \in E(S)$ or $S$ has an identity 1 and $1 \in X$, that $S$ is a $q$ partial group, and $K$ is a normal subgroup of $S_{1}$ (the maximal subgroup of $S$ with identity 1 ), then $K^{S}$ is a $q$ subpartial group of $S$. 


\section{Minimal conditions on Clifford semigroup congruences}

We also characterize the (normal) core $H_{S}$ of a wide subpartial group $H$ of a $q$ partial group $S$ in terms of the group-theoretic cores of $H_{e}$ in $S_{e}(e \in E(S)) . H_{S}$ need not be a $q$ subpartial group of $S$, whence we introduce the notion of a $q$ (normal) core showing that the $q$ core of a wide subpartial group $H$ of a $q$ partial group $S$ is precisely $\mathbf{Q}\left(H_{S}\right)$.

In Section 3, we show that a $q$ partial group $S$ satisfies min $-q n$ (the minimal condition on $q$ normal subpartial groups) if and only if $S_{1}$ satisfies $\min -n$. Introducing the notions of finite index and local finite index in partial groups (with identities), we show that $\min -q n$ in $q$ partial groups is inherited by $q$ subpartial groups with local finite indexes extending Theorem 1.8.

Whereas for partial groups with identities, we show that $\min -n$ implies $\min -q n$ for wide subpartial groups with finite indexes.

These two results can be formulated in terms of $(q)$ congruences. In particular, the former result may have the version.

In $q$ partial groups, the minimal condition on $q$ congruences is inherited by $q$ subpartial groups with local finite indexes.

\section{Normal closures and cores}

Given a partial group $S$ and a nonempty subset $X$ of $S$, we define the normal closure of $X$, denoted by $X^{S}$, to be the intersection of all normal subpartial groups of $S$ containing $X$. Evidently, $X^{S}$ is the smallest normal subpartial group of $S$ containing $X$. Analogous to the known characterization of normal closures in groups (see Section 1), we have the following lemma.

Lemma 2.1. Let $S$ be a partial group and let $\varnothing \neq X \subset S$.

(i) If $X \cap S_{e} \neq \varnothing$ for all $e \in E(S)$, then

$$
X^{S}=\left\langle s^{-1} X s: s \in S\right\rangle .
$$

In particular, if $H$ is a wide subpartial group of $S$, then

$$
H^{S}=\left\langle s^{-1} H s: s \in S\right\rangle \text {. }
$$

(ii) If S has an identity 1 and $1 \in X$, then

$$
X^{S}=\left\langle s^{-1} X s: s \in S\right\rangle .
$$

Proof. (i) Clearly, $\left\langle s^{-1} X s: s \in S\right\rangle$ is a subpartial group of $S$ containing $X$, and by the hypothesis, it is also wide. Let $s \in S$ and let $y \in\left\langle s^{-1} X s: s \in S\right\rangle$ be a generator, say $y=$ $s_{1}^{-1} x s_{1}$, for some $s_{1} \in S, x \in X$. Then $s^{-1} y s=s^{-1} s_{1}^{-1} x s_{1} s=\left(s_{1} s\right)^{-1} x\left(s_{1} s\right) \in\left\langle s^{-1} X s: s \in S\right\rangle$. If $y_{1}, \ldots, y_{n}$ are generators in $\left\langle s^{-1} X s: s \in S\right\rangle$, then

$$
\begin{aligned}
s^{-1}\left(y_{1} y_{2} \cdots y_{n}\right) s & =s^{-1} y_{1} s s^{-1} y_{2} s s^{-1} \cdots s s^{-1} y_{n} s \\
& =\left(s^{-1} y_{1} s\right)\left(s^{-1} y_{2} s\right) \cdots\left(s^{-1} y_{n} s\right) \in\left\langle s^{-1} X s: s \in S\right\rangle .
\end{aligned}
$$

Hence, $\left\langle s^{-1} X s: s \in S\right\rangle$ is a normal subpartial group of $S$ containing $X$. Suppose that $K$ is a normal subpartial group of $S$ and that $X \subset K$. Then for any $s \in S, x \in X, s^{-1} x s \in K$ 
(since $x \in K$ ), and so $\left\langle s^{-1} X s: s \in S\right\rangle \subset K$. Therefore, $\left\langle s^{-1} X s: s \in S\right\rangle$ is the normal closure of $X$ and (i) is proved.

(ii) For any $e \in E(S), e=e^{-1} 1 e \in\left\langle s^{-1} X s: s \in S\right\rangle$. Thus $\left\langle s^{-1} X s: s \in S\right\rangle$ is a wide subpartial group of $S$ containing $X$. The result follows as in (i).

Lemma 2.2. Let $S$ be a q partial group and let $K$ be a normal subgroup of $S_{1}$. Then $K^{S}$ is a $q$ normal subpartial group of $S$.

Proof. Let $H=K^{S}$. By Lemma 2.1(ii), $H=\left\langle s^{-1} K s: s \in S\right\rangle$. Thus, it is sufficient to show that $H_{e} \subset H_{1} e$ for all $e \in E(S)$ (which implies that $\varphi_{1, e}: H_{1} \rightarrow H_{e}$ is an epimorphism for all $e \in E(S)$ ). Let $e \in E(S)$ be fixed but arbitrary, and let $x \in H_{e}$. Since $K$ is a subgroup of $S_{1}$, then $x$ may be written as a product of generators $x=x_{1}^{-1} k_{1} x_{1} x_{2}^{-1} k_{2} x_{2} \cdots x_{n}^{-1} k_{n} x_{n}$ for some $x_{i} \in S$ and $k_{i} \in K$, and $i=1, \ldots, n$. We have $e_{k_{i}}=1$ for all $i \in I$, and so

$$
e=e_{x}=e_{x_{1}} e_{x_{2}} \cdots e_{x_{n}} .
$$

Since $S$ is a $q$ partial group, we have

$$
x_{i}=s_{i} e_{x_{i}}
$$

for some $s_{i} \in S_{1}, i=1,2, \ldots, n$. Thus

$$
x=s_{1}^{-1} k_{1} s_{1} s_{2}^{-1} k_{2} s_{2} \cdots s_{n}^{-1} k_{n} s_{n} e_{x_{1}} e_{x_{2}} \cdots e_{x_{n}} .
$$

Since $K$ is normal in $S_{1}, k_{i}^{\prime}=s_{i}^{-1} k_{i} s_{i} \in K, i=1,2, \ldots, n$, and we have

$$
x=k_{1}^{\prime} k_{2}^{\prime} \cdots k_{n}^{\prime} e=k e, \quad \text { where } k=k_{1}^{\prime} k_{2}^{\prime} \cdots k_{n}^{\prime} \in K
$$

and so $x \in K e$. Clearly, $H_{1}=\left(K^{S}\right)_{1}=\left\langle s^{-1} K s: s \in S_{1}\right\rangle=K$. Thus $x \in H_{1} e$. Therefore $H_{e} \subset$ $H_{1} e$.

The notion of a core (or normal interior) in groups can be also extended to partial groups. Let $S$ be a partial group and let $H$ be a wide subpartial group of $S$. The core (normal interior) of $H$ in $S$, denoted by $H_{S}$, is the join of all normal subpartial groups of $S$ contained in $H$. In other words, $H_{S}$ is the largest normal subpartial group of $S$ contained in $H$. Cores in $q$ partial groups can be characterized in terms of cores in groups. We have the following theorem.

Theorem 2.3. Let $S$ be a q partial group and let $H$ be a wide subpartial group of $S$. Then $\left(H_{S}\right)_{e}$ is the group-theoretic core of $H_{e}$ in $S_{e}$ for every $e \in E(S)$, that is, $H_{S}$ is a semilattice of groups

$$
H_{S}=\left[E(S), K_{e}, \varphi_{e, f}\right]
$$

where $K_{e}$ is the core of the subgroup $H_{e}$ in $S_{e}, e \in E(S)$.

Proof. By hypothesis, $K=\bigcup_{e \in E(S)} K_{e}$ is a union of disjoint groups indexed by the semilattice $E(S)$. By Fact 1.10, for every $e \in E(S), K_{e}=\bigcap_{s \in S_{e}} s^{-1} H_{e} s$. For $e \geq f$ in $E(S)$, define $\varphi_{e, f}: K_{e} \rightarrow K_{f}$ by $x \mapsto x f\left(x \in K_{e}\right)$. To show that $K$ is a semilattice of groups, it is sufficient to show that $\varphi_{e, f}$ is well defined in the sense that $\varphi_{e, f}\left(K_{e}\right) \subset K_{f}$ for all $e \geq f$ in $E(S)$. 
Thus, let $x \in K_{e}=\bigcap_{s \in S_{e}} s^{-1} H_{e} s$. We must show that $x f \in K_{f}=\bigcap_{s \in S_{f}} s^{-1} H_{f} s$. For this, let $s$ be an arbitrary element of $S_{f}$. Since (by hypothesis) $\varphi_{e, f}: S_{e} \rightarrow S_{f}$ is an epimorphism, there exists $s_{1} \in S_{e}$ such that $s=s_{1} f$. Now, $x \in \bigcap_{s \in S_{e}} s^{-1} H_{e} s$ implies that $x=s_{1}^{-1} y s_{1}$, for some $y \in H_{e}$. Whence, $x f=\left(s_{1} f\right)^{-1}(y f)\left(s_{1} f\right) \in s^{-1} H_{f} s$. Since $s$ is arbitrary in $S_{f}$, we obtain $x f \in \bigcap_{s \in S_{f}} s^{-1} H_{f} s=K_{f}$. Hence $K$ is a (strong) semilattice of groups. It follows that $K$ is a subpartial group of $S$ which is clearly wide. To complete the proof, we have to show that $H_{S}=K$. Thus it is sufficient to show that $K$ is normal in $S$, that $K \subset H$, and that $K$ is the largest with respect to this property. That $K \subset H$ follows trivially from the definition of $K$. To show that $K$ is normal in $S$, let $x \in S$ and let $y \in K$, say $x \in S_{e}, y \in K_{f}$, for some $e, f \in E(S)$. Thus, $x y=x y e f$, and we have $x^{-1} y x=(x f)^{-1}(y e f)(x f)$. Clearly, $x f \in S_{e f}$ and $y e f \in K_{e f}$. By the construction of $K, K_{e f}$ is normal in $S_{e f}$. It follows that $x^{-1} y x \in K_{e f} \subset K$. Thus $K$ is a normal subpartial group of $S$. Finally, let $N$ be a normal subpartial group of $S$ such that $N \subset H$. We have for every $e \in E(S), N_{e}$ is normal in $S_{e}$ and $N_{e} \subset H_{e}$. Since $K_{e}$ is the core of $H_{e}$ in $S_{e}$, we obtain $N_{e} \subset K_{e}$. Therefore $N \subset K$.

Let $S$ be a partial group with 1 and let $H$ be a wide subpartial group of $S$. We define the $q$ core of $H$ in $S$ to be the join of all $q$ normal subpartial groups of $S$ contained in $H$.

THEOREM 2.4. If $S$ is a q partial group and $H$ is a wide subpartial group of $S$, then the q core of $H$ in $S$ is $\mathbf{Q}\left(H_{S}\right)$, where $H_{S}$ is the core of $H$ in $S$.

Proof. We have $H_{S}=\langle N: N \triangleleft S, N \subset H\rangle$, let $\mathbf{N}_{H}$ be the family of all normal subpartial groups $N$ of $S$ contained in $H$. Then $H_{S}=\left\langle N: N \in \mathbf{N}_{H}\right\rangle$. Let $q \mathbf{N}_{H}$ be the family of all $q$ normal subpartial groups of $S$ contained in $H$, we have $K \in q \mathbf{N}_{H}$ if and only if $K=\mathbf{Q}(N)$, for some $N \in \mathbf{N}_{H}$, that is, $q \mathbf{N}_{H} \subset\left\{\mathbf{Q}(N): N \in \mathbf{N}_{H}\right\}$. On the other hand, if $N \in \mathbf{N}_{H}$, then, since $\mathbf{Q}(N)$ is normal in $S[1]$, we have $\mathbf{Q}(N) \in q \mathbf{N}_{H}$. Thus, $\left\{\mathbf{Q}(N): N \in \mathbf{N}_{H}\right\} \subset q \mathbf{N}_{H}$. Then, $q \mathbf{N}_{H}=\left\{\mathbf{Q}(N): N \in \mathbf{N}_{H}\right\}$. By definition and [1, Lemma 4.2], we have the $q$ core of $H$ in $S$ which is

$$
\begin{aligned}
\left\langle K: K \in q \mathbf{N}_{H}\right\rangle & =\left\langle\mathbf{Q}(N): N \in \mathbf{N}_{H}\right\rangle \\
& =\mathbf{Q}\left(\left\langle N: N \in \mathbf{N}_{H}\right\rangle\right)=\mathbf{Q}\left(H_{S}\right) .
\end{aligned}
$$

\section{Minimal conditions on $q$ congruences}

We use the machinary that has been developed so far to extend a result in groups concerning the inheritance of minimal condition on normal subgroups of a given group to subgroups with finite indexes, and obtain analogous results for partial groups and $q$ partial groups.

Recall that a partially ordered set $(P, \leq)$ is said to satisfy the minimal condition if any nonempty subset of $P$ contains a minimal element. This is equivalent to saying that $P$ satisfies the descending chain condition: there does not exist an infinite properly descending chain $x_{1}>x_{2}>\cdots$ in $P$.

In particular, a group $G$ satisfies $\min -n$ if $(\mathbf{N}(G), \subset)$ satisfies the minimal condition, where $\mathbf{N}(G)$ is the set of all normal subgroups of $G$. Analogously, we say that a partial group $S$ satisfies $\min -n$ if $(\mathbf{N}(S), \subset)$ satisfies the minimal condition. Let $S$ be a partial group with identity 1, we say that $S$ satisfies $\min -q n$ if $(\mathbf{Q N}(G), \subset)$ satisfies the minimal 
condition, or equivalently if there does not exist an infinite properly descending chain $K_{1} \supset K_{2} \supset \cdots$ in $\mathbf{Q N}(S)$. Here $\mathbf{N}(S)$ and $\mathbf{Q N}(S)$ are defined as in Section 1.

Lemma 3.1. Let $S$ be a partial group with identity 1 .

(i) If $S$ satisfies $\min -n$, then $S_{1}$ satisfies $\min -n$.

(ii) If $S_{1}$ satisfies $\min -n$, then $S$ satisfies $\min -q n$.

Proof. (i) Suppose that $S$ satisfies $\min -n$ but $S_{1}$ does not. There exists an infinite properly descending chain

$$
N_{1} \supset N_{2} \supset \cdots \quad \text { in } \mathbf{N}\left(S_{1}\right)
$$

For each $i=1,2, \ldots, N_{i}^{S}$ (the normal closure of $N_{i}$ in $S$ ) is a normal subpartial group of $S$ and we have a descending chain

$$
N_{1}^{S} \supset N_{2}^{S} \supset \cdots \quad \text { in } \mathbf{N}(S) .
$$

By $\min -n$ of $S$, we obtain

$$
N_{j}^{S}=N_{j+1}^{S} \quad \text { for some } j .
$$

Since $N_{j} \supset N_{j+1}$ and $N_{j} \neq N_{j+1}$, there exists some element $s \in N_{j}$ with $s \notin N_{j+1}$. We have $s \in N_{j}^{S}=N_{j+1}^{S}$. Thus, $s$ may be written as an expansion,

$$
s=y_{1}^{-1} x_{1} y_{1} y_{2}^{-1} x_{2} y_{2} \cdots y_{n}^{-1} x_{n} y_{n},
$$

with $y_{i} \in S, x_{i} \in N_{j+1}, i=1,2, \ldots, n$. Since $e_{s}=1$, we must have $e_{y_{i}}=1$ for all $i=1,2, \ldots, n$, that is, $y_{i} \in S_{1}$ for all $i=1,2, \ldots, n$. Now, $N_{j+1}$ being a normal subgroup of $S_{1}$ implies that $y_{i}^{-1} x_{i} y_{i} \in N_{j+1}$, for all $i=1,2, \ldots, n$. Therefore, $s \in N_{j+1}$, a contradiction.

(ii) Suppose that $S_{1}$ satisfies $\min -n$ but $S$ does not satisfy $\min -q n$. There exists an infinite properly descending chain

$$
K_{1} \supset K_{2} \supset \cdots \quad \text { in } \mathbf{Q N}(S) .
$$

For each $i=1,2, \ldots,\left(K_{i}\right)_{1}$ is a normal subgroup of $S_{1}$, where $\left(K_{i}\right)_{1}$ is the maximal subgroup of $K_{i}$ with identity 1 . Thus, the above chain induces a descending chain

$$
\left(K_{1}\right)_{1} \supset\left(K_{2}\right)_{1} \supset \cdots \quad \text { in } \mathbf{N}\left(S_{1}\right) .
$$

Since $S_{1}$ satisfies $\min -n$, we have for some $j$

$$
\left(K_{j}\right)_{1}=\left(K_{j+1}\right)_{1} .
$$

By assumption, $K_{j} \supset K_{j+1}$ and $K_{j} \neq K_{j+1}$. Thus we must have, for some $e \in E(S)$,

$$
\left(K_{j}\right)_{e} \supset\left(K_{j+1}\right)_{e}, \quad\left(K_{j}\right)_{e} \neq\left(K_{j+1}\right)_{e} .
$$

There exists $x \in\left(K_{j}\right)_{e}$ such that $x \notin\left(K_{j+1}\right)_{e}$. Since $K_{j}$ is a $q$ partial group, we have $x=s e$ for some $s \in\left(K_{j}\right)_{1}=\left(K_{j+1}\right)_{1}$. Now, $s \in\left(K_{j+1}\right)_{1}$, which implies that $s e \in\left(K_{j+1}\right)_{e}$ and so $x \in\left(K_{j+1}\right)_{e}$, a contradiction. 
Using Lemmas 3.1(ii), 2.2, and proceeding as in the proof of Lemma 3.1(i), we obtain the following lemma.

Lemma 3.2. A q partial group $S$ satisfies $\min -q n$ if and only if $S_{1}$ satisfies $\min -n$.

Let $S$ be an arbitrary partial group. A wide subpartial group $H$ of $S$ is said to have a finite index in $\mathrm{S}$ if $S=H T$, for some finite subset $T$ of $S$.

Lemma 3.3. Let $S$ be a partial group and let $H$ be a wide subpartial group of $S$. If $H$ has a finite index in $S$, then $H_{e}$ has a finite index in $S_{e}$ for every e $\in E(S)$.

Proof. By assumption, $S=H T$, for some finite subset $T$ of $S$. Let $e \in E(S)$ be fixed but arbitrary. For each $x \in S_{e}$, we have $x \in S_{e} \subset S=H T$. Thus $x=h t_{x}$, for some $h \in H$ and $t_{x} \in T$. We have, $e=e_{x}=e_{h} e_{t_{x}}$, and so $e \leq e_{h}$ and $e \leq e_{t_{x}}$, that is, $e e_{h}=e$ and $e e_{t_{x}}=e$. Now, $x=h t_{x}=(h e) t_{x} \in H_{e} t_{x}=H_{e}\left(e t_{x}\right)$. Setting ${ }_{x} T=\left\{e t_{x}: x \in S_{e}\right\}$, then clearly ${ }_{x} T$ is a finite subset of $S_{e}$ and $S_{e} \subset H_{e x} T$. Also, $H_{e x} T \subset S_{e} S_{e}=S_{e}$. Therefore, $S_{e}=H_{e x} T$, which proves that $H_{e}$ has a finite index in $S_{e}$.

Let $S$ be a partial group with identity 1 and let $H$ be a wide subpartial group of $S$. We say that $H$ has a local finite index in $S$ if $H_{1}$ has a finite index in $S_{1}$. Clearly, by Lemma 3.3, for a wide subpartial group $H$ of a partial group $S$ with identity, we have a finite index in $S$ which implies a local finite index in $S$.

For $q$ partial groups, the implication of Lemma 3.3 may be refined as follows.

Lemмa 3.4. Let $S$ be a q partial group and let $H$ be a wide subpartial group of $S$. If $H$ has a local finite index in $S$, then $H_{e}$ has a finite index in $S_{e}$ for every e $\in E(S)$.

Proof. We have $S_{1}=H_{1} T$, for some finite subset $T \subset S_{1}$. Let $e \in E(S)$, since $S$ is a $q$ partial group, then $S_{e}=S_{1} e$. Thus

$$
S_{e}=H_{1} T e=\left(H_{1} e\right)(T e)=\left(H_{1} e\right)_{e} T,
$$

where ${ }_{e} T$ is the finite set $T e \subset S_{e}$. Since $H$ is wide, $H_{1} e \subset H$, in particular, $H_{1} e \subset H_{e}$. Therefore,

$$
S_{e}=\left(H_{1} e\right)_{e} T \subset H_{e} T \subset S_{e}
$$

Thus, $S_{e}=H_{e} e^{T}$ and the result follows.

Now we give our main result which is an extension of [6, Theorem 3.1.8].

THeORem 3.5. If a q partial group $S$ satisfies min-qn and $H$ is a $q$ subpartial group of $S$ with local finite index, then $H$ satisfies $\min -q n$.

Proof. By definition (of local finite index), $H_{1}$ has a finite index in $S_{1}$, and by Lemma 3.2, $S_{1}$ satisfies $\min -n$. Since $H_{1}$ is a subgroup of $S_{1}$, Wilson theorem [6, Theorem 3.1.8] implies that $H_{1}$ satisfies $\min -n$. Again by Lemma 3.2, we have $H$ satisfying $\min -q n$.

For partial groups with identities, we have the following version of Theorem 3.5.

THeORem 3.6. If a partial group $S$ with identity satisfying $\min -n$ and $H$ is a wide subpartial group of $S$ with finite index, then $H$ satisfies min $-q n$. 
The proof follows similarly by applying Lemmas 3.1(i), 3.3, [6, Theorem 3.1.8], and Lemma 3.1(ii).

In view of Theorems 1.7 and 1.1, the above two Theorems (3.5 and 3.6) can be formulated in terms of $q$ congruences and idempotent-separating congruences, respectively. For instance, by Theorems 1.7 and 3.5, we have the following corollary.

Corollary 3.7. If a q partial group $S$ satisfies the minimal condition on $q$ congruences and $H$ is a $q$ subpartial group of $S$ with local finite index, then $H$ satisfies the minimal condition on q congruences.

\section{References}

[1] M. E.-G. M. Abdallah, L. N. Gab-Alla, and S. K. M. Elagan, On semilattices of groups whose arrows are epimorphisms, to appear in International Journal of Mathematics and Mathematical Sciences.

[2] A. M. Abd-Allah and M. E.-G. M. Abdallah, Congruences on Clifford semigroups, Pure Mathematics Manuscript 7 (1988), 19-35.

[3] __ On Clifford semigroups, Pure Mathematics Manuscript 7 (1988), 1-17.

[4] J. M. Howie, An Introduction to Semigroup Theory, Academic Press, London, 1976.

[5] M. Petrich, Inverse Semigroups, Pure and Applied Mathematics (New York), John Wiley \& Sons, New York, 1984.

[6] D. J. S. Robinson, A Course in the Theory of Groups, Graduate Texts in Mathematics, vol. 80, Springer, New York, 1982.

M. El-Ghali M. Abdallah: Department of Mathematics, Faculty of Science, Menoufiya University, Shebin El-kom 32511, Egypt

E-mail address: mohamed_elghaly@yahoo.com

L. N. Gab-Alla: Department of Mathematics, Faculty of Science, Menoufiya University,

Shebin El-kom 32511, Egypt

E-mail address: layla_nashed2006@yahoo.com

Sayed K. M. Elagan: Department of Mathematics, Faculty of Science, Menoufiya University,

Shebin El-kom 32511, Egypt

E-mail address: sayed_khalil2000@yahoo.com 


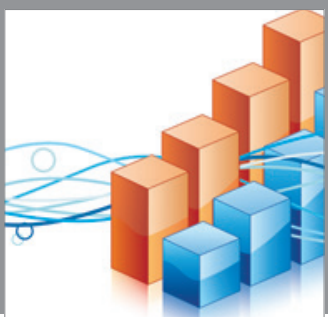

Advances in

Operations Research

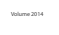

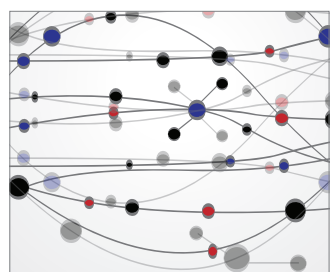

\section{The Scientific} World Journal
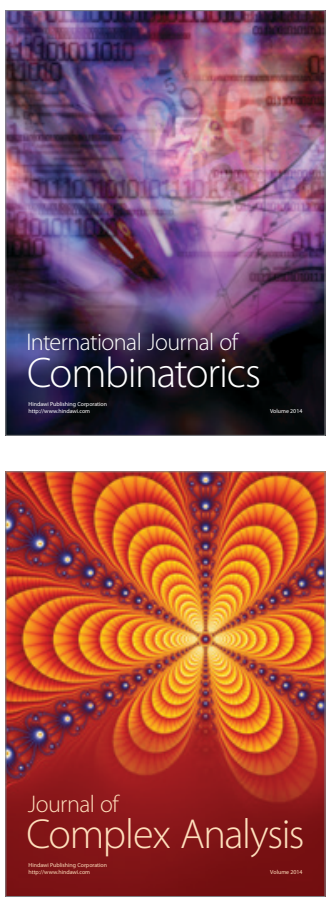

International Journal of

Mathematics and

Mathematical

Sciences
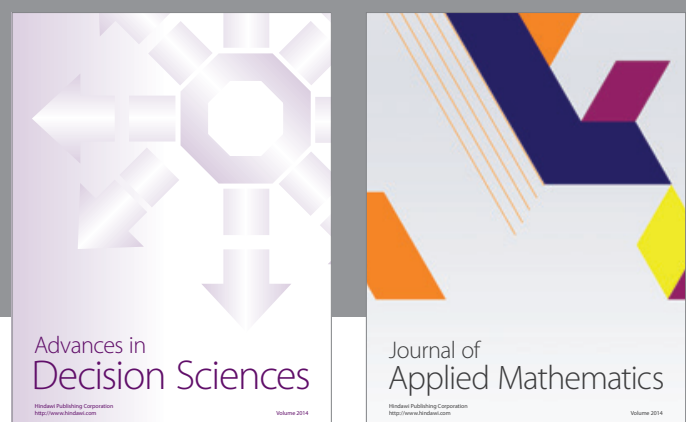

Journal of

Applied Mathematics
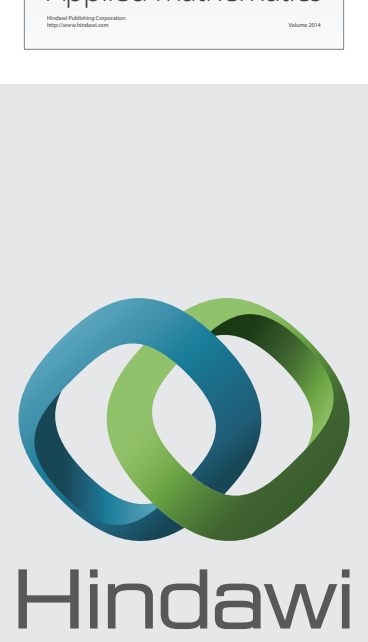

Submit your manuscripts at http://www.hindawi.com
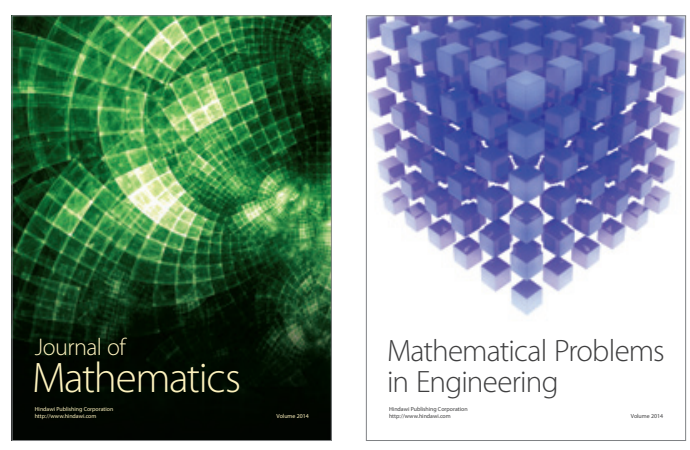

Mathematical Problems in Engineering
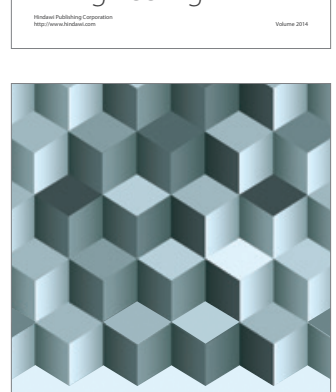

Journal of

Function Spaces
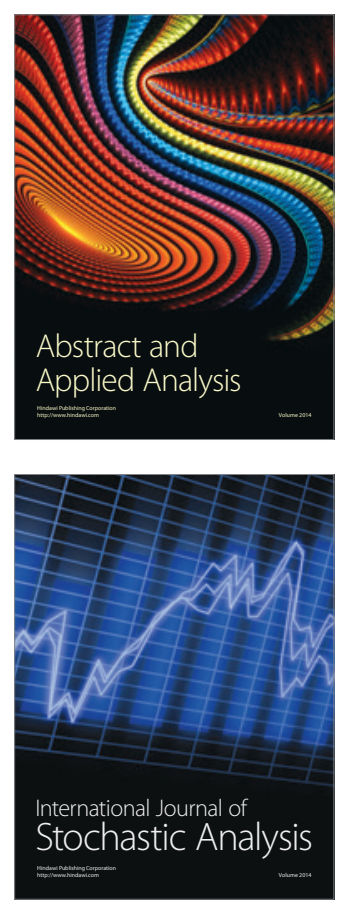

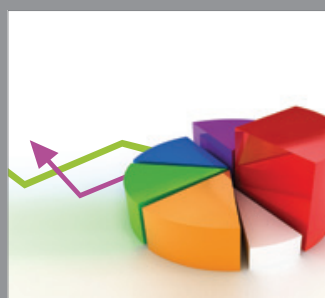

ournal of

Probability and Statistics

Promensencen
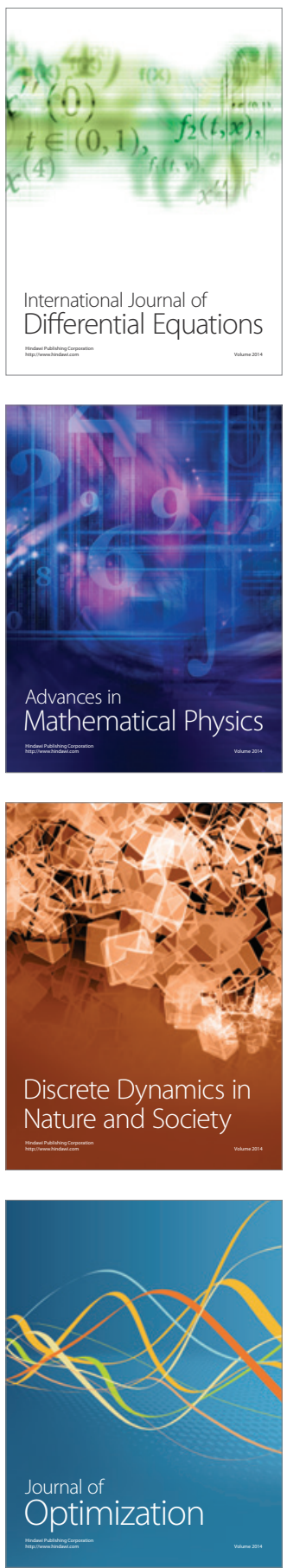